\title{
Back in control
}

Spinal cord injury disrupts the pathway between brain and muscle, causing paralysis. One potential strategy for treatment is to use a brain-machine interface to route control signals from the brain directly to the muscles, bypassing the site of injury. For the first time, Moritz and colleagues have shown that an artificial device can compensate for paralysis in monkeys.

The study combined previous lines of research which showed that paralysed muscles can be activated by functional electronic stimulation (FES) and that motor cortex neurons can control computer cursors and robotic arms through a brainmachine interface. Single-cell activity in the motor cortex was converted to functional stimulation of paralysed wrist muscles in two monkeys. The monkeys could then learn to control this activity to produce goal-directed muscle contractions.

To determine whether neuronal activity could be used to stimulate muscle directly, the authors temporarily paralysed peripheral nerves innervating the wrist with a local anaesthetic. Neuronal activity was then converted into FES of the paralysed muscle using a computer interface, and the position of a cursor on a computer screen was set to represent FES-induced wrist torque. The monkeys quickly learned to control their wrist muscles using only the selected neuron's activity, and so were able to produce the necessary wrist torque to move the cursor to targets and receive a reward. They were also able to relax the muscle when required, by keeping the neuron's firing rate below a given threshold.

The monkeys achieved high task accuracy after only a few minutes of practice with 44 out of 45 neurons tested. Task success was independent of the original direction selectivity of the neuron, suggesting that potentially any neuron in motor areas of the brain could be used. The system was also flexible, with the monkeys able to achieve different levels of torque strength by controlling the activity of just a single neuron. Additionally, the authors showed that a single neuron could be used to simultaneously control two sets of antagonist muscles to enable bidirectional movement, and one of the monkeys could control two cell-muscle pairs simultaneously.

This study indicates that flexible, goal-directed movement can be achieved with a relatively simple single-cell-FES interface. With further development, this strategy might eventually lead to implantable devices enabling some volitional movement in individuals living with paralysis.

Catherine Sebastian

ORIGINAL RESEARCH PAPER Moritz, C. T. et al. Direct control of paralysed muscles by cortical neurons. Nature 15 Oct 2008 (doi:10.1038/ nature07418) FURTHER READING Velliste, M. et al. Cortical control of a prosthetic arm for self-feeding. Nature 453, 1098-1101 (2008) 